ENHANCED BONDING IN THE HIGH RISK NEONATE.
91 Barbara M. Ostfeld, Richard $H$. Smith, Kathy $G$. Blannett, (Sponsored by Thomas Hegyi), UMDNJ-Rutgers Medical Schoot, Monmouth Medical Center, Department of Pediatrics Long Branch, New Jersey

An intervention program designed to enhance maternal infant bonding in high risk dyads significantly improved maternal child behavior and interaction. The preterm neonate, whose appearance, behavior and prognos is violate maternal expectations, has disrupted mother-infant relationship because of diminished contact, erratic responsivity and stressed maternal emotions.

Thirty four mothers of high risk infants (BW $1630+330 \mathrm{~g}$, GA $32+2$ wks, Parmalee risk factor $77.5+12.3$ ) comprised the study group, and were exposed to videotapes designed to reduce emotional crises, increase confidence, and enhance ability to interact with the infant. Mother infant contact, discussion, and demonstrations followed each session. Controls consisted of mothers of 35 infants (BW $1590 \pm 380 \mathrm{~g}$, GA $32+2$, Parmalee risk factor $72.5+15.3$ ), exposed to conventional communication.

EvaTuation with a behavioral rating scale was performed one month post discharge $(G A 40+1 \mathrm{wk})$. The results demonstrated increased maternal attention $(P 0.01)$, maternat responsivity ( $P$ O. Creased maternal attention maternal infant mutuality ( $P$, 0.05$)$ in the study group. Repeat examination at 8 months post discharge showed increased smiling behavior ( $P$ 0.01) in study infants. Significant inverse correlations were noted between the effects of intervention and socioeconomic status. In these mother-infant dyads improved relationships were achieved by a specifically designed program.

IMPACT OF TRANSCUTANEOUS OXYGEN MONITORING (TCOM) ON $\dagger 92$ QUALITY AND COST OF NEONATAL INTENSIVE CARE. Keith J. Univ. of South Alabama, Depts. of Ped. and Resp. Therapy, Mobile, AL, 36617 .

Technological advances in health care equipment have parallel effects on quality and cost of patient care. We studied the impact of TCOM on patient care and patient costs in our ICN over a 5 year period (1978-1982). The number of patient days (PD), blood gases (BG), and hours of TCOM were ascertained for each year, and used to derive yearly cost data for $B G$ analysis and TCOM, and to estimate BG cost had TCOM not been available (using $B G / P D$ for 1978 , cost of $\$ 45.25 / B G$, and cost of $\$ 4.25 /$ TCOM hr.).

$\begin{array}{clccccc}\text { Year } & \text { BG/PD } & \text { TCOM/PD } & \begin{array}{c}\text { BG } \\ \left(\$ \times 10^{3}\right)\end{array} & \begin{array}{c}\text { TCOM } \\ \left(\$ \times 10^{3}\right)\end{array} & \begin{array}{c}\text { BGSTCQM } \\ \left(\$ \times 10^{3}\right)\end{array} & \begin{array}{c}\text { Saving } \\ \left(\$ \times 10^{3}\right)\end{array} \\ 1978 & 2.13 & 0 & 470 & 0 & 470 & 0 \\ 1979 & 2.16 & .78 & 577 & 19.6 & 595 & -1 \\ 1980 & 1.88 & 1.92 & 540 & 51.6 & 635 & +43 \\ 1981 & 1.71 & 3.00 & 471 & 77.8 & 618 & +64 \\ 1982 & 1.28 & 3.45 & 400 & 101.4 & 697 & +196\end{array}$

$\begin{array}{lllllll}1982 & 1.28 & 3.45 & 400 & 101.4 & 697 & +196\end{array}$ The table shows a substantial fall in BG/PD concomitant with the increase in TCOM/PD. Based on estimates of $B G$ costs if TCOM had not been available, costs of patient care fell by almost 200,000 by the fourth year (1982) of TCOM. Excluding capital expenditures for BG and TCOM equipment, hospital costs durtal expenditures for BG and TCOM equipment, hospital costs during implementation of TCOM fell by a net $\$ 6240$. We conclude that TCOM offers the potential for (1) better patient management with
fewer invasive procedures, (2) lower patient costs, (3) no significant increase in hospital expenditures.

STATE, POSTURE AND MOTOR ACTIVITY IN THE VERY LOW

93 BIRTH WEIGHT NEONATE。 rvette Peña, Annabel Teberg (Spon. by Paul Y.K. Wu). Univ. of So. Calif. Sch. Med., Los Angeles County-USC Med. Ctr., Dept. of Peds., L.A.
Eleven preterm infants with gestational age (GA) ranging from Eleven preterm infants with gestational age (GA) ranging from
24 to 34 weeks were observed during the neonatal period to deter mine state, posture, quantity and quality of motor activity, and any effect of ventilatory assistance (VA) upon these functions for the 7 VA infants in the group. Infants were observed for a 10 minute period at a specific time daily during the first week, then weekly throughout the nursery course. State was detexmined using Brazelton grading. Posture was recorded at the beginning using Brazelton grading. Posture was and limbs of the observation. All movements of head, eyes, body and limbs were recorded according to defined categories. The predominant behavioral states observed were light sleep and dozing. No specific preference for body and/or limb posture was observed. All infants had frequent twitches and movements predominantly of the limbs which produced posture changes. Quantity of movements did not follow any particular patterns. However, a marked change in quantity of movements was usually associated with sedation and/or illness. Clonus and tremors were almost always seen in the ventilatory assisted infant, and rarely the non-respirator infants. Posture as well as quantity and quality of movements was not related to GA or conceptual age (CA). In conclusion, the serial and systematic observation of motor activity in the preterm infant confirms the time honored clinical observation that changes in motor activity may herald significant illness. In spite of previous work by others, in this group of preterm infants posture preference did not relate to $\mathrm{GA}$ or $\mathrm{CA}$ 。
DENTITION IN THE LOW BIRTHWEIGHT INFANT. Janice

94 Pimlott, Pamela Fitzhardinge, Tom Howley and Gordon and Pediatrics, Hospital for Sick Children, Toronto.

Two hundred twenty seven infants with birthweights $<1501 \mathrm{~g}$ showed a similar timing and pattern of primary tooth eruption as reported for normal full term infants. The average number of erupted teeth at the post-term age of $6,9,12$, and 18 months were $0.5 \pm 1.0,2.9 \pm 2.2,5.8 \pm 2.3$, and $11.6 \pm 3.6$ respectively. There was no sex difference. The 40 IUGR infants had significantly fewer teeth at 9 and 18 months post-term than the rest of the sample $(p<0,05)$. The results suggest that the timing of tooth eruption is determined by post-conceptional age but may be delayed by prolonged malnutrition.

Enamel development in primary teeth was studied in another sample of 106 children <1501g birthweight at ages 2 to 8 years. $37 \%$ showed hypoplasia in the maxillary anterior teeth located predominantly in the incisal half. Less than 10\% of the other teeth were affected. Similar lesions have been reported in $1-9 \%$ of full term children. Permanent incisors were present in 37 children: 21 (58\%) showed areas of enamel hypocalcification compared with 3 of 40 full term controls. The location of both the hypoplasia in primary tooth enamel and the hypocalcification in the permanent enamel suggest a neonatal origin specific to prematurity, but no statistical relationship could be shown with prematurity, but no statistical relationship could be shown
the degree of prematurity, IUGR or neonatal calcium levels.
A COMPUTER BASED STATEWIDE PERINATAL NETWORK. Ronald

$95 \frac{\text { L. Poland, Robert } 0 \text {. Bollinger, Glenn E. Cummings, Depts }}{\text { of Pediatrics and Oncology, Wayne State University } \mathcal{E}}$ Children Hospital of Michigan, Detroit.

We have developed a statewide network (PAM/NET) using a large mainframe, time-sharing computer which provides perinatal and follow-up databases and flexible report generation along with an on-line forum for 20 widely separated tertiary care perinatal units in Michigan. The data bases manage discharge summary information from neonatal special care units (about 5000 infants per year) and from visits to Developmental Assessment (follow-up) $\mathrm{Clin-}$ ic. Each unit manages and controls its own data system. However, pooling and sharing of data is facilitated since all of the sites in the network share the same hardware and sof tware. The system converts the data entered into prosaic summary letters which serve as hospital discharge summaries and as dictated letters to referring physicians. The cost is substantially less than that of referring physicians. The cost generated in the tradional way. The users are provided summaries generated in the traditional way. The users are provided
with a simple but powerful interactive query language which allwith a simple but powerful interactive query language which all-
ows the local participants to analyze their own data and to conduct their own research.

In addition, the users of the data system are enrolled in an electronic forum which is used to address the technical problems they encounter and to provide a medium for exchange of ideas athey the participants. This forum is an on-line conferencing promong the partows for public announcements, open on-going disgram which allows for public announcements

Users with little prior computer experience have become able to use the system after a one-day training session.

\section{MANPOWER REQUIREMENTS IN PEDIATRIC INTENSIVE} CARE. Murray M. Pollack (Spon. by Glenn Rosenquist), George Wash Univ Sch of Med, Childr Hosp Nat Med Cntr, Departs of Pediatrics and Anesth, Washington, D.C.

This study 1) measured manpower requirements for patient care in the pediatric ICU and 2) correlated manpower needs with measures of severity of illness (Physiologic Stability Index: PSI) and quantity of care (Therapeutic Intervention Scoring System: TISS) to assess their usefulness in predicting staffing needs. Methods: 9 randomly selected observation shifts ( 8 hours) were studied. Manpower needs were measured by an intermittent time sampling technique. PSI and TISS scores were determined for the observation shift, previous shift and previous 24 hours. Results: 91 patients were observed. Total personnel previous 24 hours. Results: 91 patients were observed. Total personnel $\mathrm{hr} / \mathrm{pt}=8.1 \pm .5$ (range $.7-16.4), \mathrm{MD} \mathrm{hr} / \mathrm{pt}=1.2 \pm .2$ (range $0-10.1$ ) and
respiratory therapy $(\mathrm{RT}) \mathrm{hr} / \mathrm{pt}=0.3 \pm .1$ (range 0.2 .4 ). Correlations of manpower times with PSI and TISS scores were significant $(p<.01)$. For the observation shift these were:

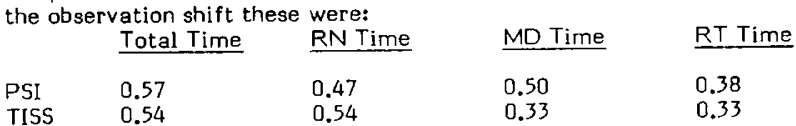

TISS 0.54 manpower times were also significantly correlated with previous shifts and previous 24 hour PSI and TISS scores. Higher with previous shifts and previous 24 hour
order curve fitting did not improve the correlations. Conclusions:
Manpower requirements for pediatric ICU's are extensive. PSI and TISS Manpower requirements for pediatric ICU's are extensive. PSI and TISS
scores which are routinely collected in many ICU's can assess trends in staffing needs but not individual patient manpower needs. 